\title{
Screening for Lung Cancer
}

\section{Diagnosis and Management of Lung Cancer, 3rd ed: American College of Chest Physicians Evidence-Based Clinical Practice Guidelines}

\author{
Frank C. Detterbeck, MD, FCCP; Peter J. Mazzone, MD, MPH, FCCP; \\ David P. Naidich, MD, FCCP; and Peter B. Bach, MD, FCCP
}

\begin{abstract}
Background: Lung cancer is by far the major cause of cancer deaths largely because in the majority of patients it is at an advanced stage at the time it is discovered, when curative treatment is no longer feasible. This article examines the data regarding the ability of screening to decrease the number of lung cancer deaths.

Methods: A systematic review was conducted of controlled studies that address the effectiveness of methods of screening for lung cancer.

Results: Several large randomized controlled trials (RCTs), including a recent one, have demonstrated that screening for lung cancer using a chest radiograph does not reduce the number of deaths from lung cancer. One large RCT involving low-dose CT (LDCT) screening demonstrated a significant reduction in lung cancer deaths, with few harms to individuals at elevated risk when done in the context of a structured program of selection, screening, evaluation, and management of the relatively high number of benign abnormalities. Whether other RCTs involving LDCT screening are consistent is unclear because data are limited or not yet mature.

Conclusions: Screening is a complex interplay of selection (a population with sufficient risk and few serious comorbidities), the value of the screening test, the interval between screening tests, the availability of effective treatment, the risk of complications or harms as a result of screening, and the degree with which the screened individuals comply with screening and treatment recommendations. Screening with LDCT of appropriate individuals in the context of a structured process is associated with a significant reduction in the number of lung cancer deaths in the screened population. Given the complex interplay of factors inherent in screening, many questions remain on how to effectively implement screening on a broader scale.
\end{abstract}

CHEST 2013; 143(5)(Suppl):e78S-e92S

Abbreviations: $\mathrm{ACCP}=$ American College of Chest Physicians; $\mathrm{CXR}=$ chest radiograph; DANTE $=$ Detection and Screening of Early Lung Cancer by Novel Imaging Technology and Molecular Essays Trial; DLCST = Danish Lung Cancer Screening Trial; LDCT = low-dose CT; NCCN = National Comprehensive Cancer Network; NLST = National Lung Screening Trial; NSCLC = non-small cell lung cancer; PLCO = Prostate, Lung, Colorectal and Ovarian Trial; $\mathrm{RCT}=$ randomized controlled trial; $\mathrm{RR}=$ rate ratio; VATS $=$ video-assisted thoracic surgery

SUMMARY OF RECOMMENDATIONS

3.2.1. In patients at risk for developing lung cancer, screening for lung cancer with chest radiograph $(\mathbf{C X R})$ once or at regular intervals is not recommended (Grade 1A).

Remark: These results should not be interpreted as diminishing the role of CXR in evaluating patients with pulmonary symptoms (an entirely different situation than screening asymptomatic individuals).

3.2.2. In patients at risk for developing lung cancer, screening for lung cancer with sputum cytology at regular intervals is not suggested (Grade 2B).

3.4.1. For smokers and former smokers who are age 55 to 74 and who have smoked for 
30 pack-years or more and either continue to smoke or have quit within the past 15 years, we suggest that annual screening with low-dose CT (LDCT) should be offered over both annual screening with CXR or no screening, but only in settings that can deliver the comprehensive care provided to National Lung Screening Trial participants (Grade $2 \mathrm{~B}$ ).

Remark: Counseling should include a complete description of potential benefits and harms, so the individual can decide whether to undergo LDCT screening.

Remark: Screening should be conducted in a center similar to those where the National Lung Screening Trial was conducted, with multidisciplinary coordinated care and a comprehensive process for screening, image interpretation, management of findings, and evaluation and treatment of potential cancers.

Remark: A number of important questions about screening could be addressed if individuals who are screened for lung cancer are entered into a registry that captures data on follow-up testing, radiation exposure, patient experience, and smoking behavior.

Remark: Quality metrics should be developed such as those in use for mammography screening, which could help enhance the benefits and minimize the harm for individuals who undergo screening.

Manuscript received September 24, 2012; revision accepted November 30, 2012.

Affiliations: From the Yale University School of Medicine (Dr Detterbeck), New Haven, CT; Respiratory Institute (Dr Mazzone), The Cleveland Clinic, Cleveland, OH; NYU Langone Medical Center (Dr Naidich), Tisch Hospital, New York, NY; and Memorial Sloan-Kettering Cancer Center (Dr Bach), New York, NY.

Funding/Sponsors: The overall process for the development of these guidelines, including matters pertaining to funding and conflicts of interest, are described in the methodology article. ${ }^{1}$ The development of this guideline was supported primarily by the American College of Chest Physicians. The lung cancer guidelines conference was supported in part by a grant from the Lung Cancer Research Foundation. The publication and dissemination of the guidelines was supported in part by a 2009 independent educational grant from Boehringer Ingelheim Pharmaceuticals, Inc.

COI grids reflecting the conflicts of interest that were current as of the date of the conference and voting are posted in the online supplementary materials.

Disclaimer: American College of Chest Physician guidelines are intended for general information only, are not medical advice, and do not replace professional medical care and physician advice, which always should be sought for any medical condition. The complete disclaimer for this guideline can be accessed at http:// dx.doi.org/10.1378/chest.1435S1.

Correspondence to: Frank C. Detterbeck, MD, FCCP, Yale School of Medicine, 330 Cedar St, PO Box 208062, New Haven, CT 06520-8062; e-mail: frank.detterbeck@yale.edu

(C) 2013 American College of Chest Physicians. Reproduction of this article is prohibited without written permission from the American College of Chest Physicians. See online for more details. DOI: $10.1378 /$ chest.12-2350
Remark: Screening for lung cancer is not a substitute for stopping smoking. The most important thing patients can do to prevent lung cancer is not smoke.

Remark: The most effective duration or frequency of screening is not known.

3.4.2. For individuals who have accumulated fewer than 30 pack-years of smoking or are either younger than age 55 or older than 74 , or individuals who quit smoking more than 15 years ago, and for individuals with severe comorbidities that would preclude potentially curative treatment and/or limit life expectancy, we suggest that CT screening should not be performed (Grade 2C).

*Recommendations 3.2.1 and 3.2.2 were approved through the American College of Chest Physicians (ACCP) Lung Cancer Guidelines (3rd ed) process. ${ }^{1}$ Recommendations 3.4 .1 and 3.4 .2 were approved through a previous multisociety guideline development process and published elsewhere ${ }^{2}$ and are included here for completeness. The majority of panel members at the ACCP Lung Cancer Guidelines (3rd ed) meeting voted in agreement with both recommendation 3.4.1 (>80\% of panelists) and 3.4.2 (>50\% of panelists); ACCP Lung Cancer Guidelines (3rd ed) recommendations require 67\% approval as described more fully in the methodology article. ${ }^{1}$

A lthough lung cancer has a similar incidence as ther common cancers (ie, breast, prostate, and colorectal), it causes 3 to 4 times as many deaths. ${ }^{3}$ This discrepancy is often attributed in part to the fact that screening for breast, prostate, and colon cancer has been commonly practiced, whereas it is not for lung cancer. This article focuses on chest radiographs (CXRs), sputum analysis, and low-dose CT (LDCT) imaging as screening tests for lung cancer because these have been addressed by large randomized trials. Other tests such as blood tests, autofluorescence bronchoscopy, or exhaled breath analysis are not addressed because they are under development and have not yet been evaluated in randomized trials assessing a mortality benefit.

\subsection{Methods}

The following questions were selected as being most relevant. All pertain to asymptomatic, otherwise healthy adults with no history of lung cancer who are at an elevated risk for lung cancer (see also Table $1 \mathrm{~S}$ ):

- What is the rate of death from lung cancer (ie, lung cancer mortality) among individuals at elevated risk of lung cancer who undergo screening with LDCT compared with either no screening or screening with another modality?

- What is the rate of death or complications resulting from biopsies of detected lesions among individuals at elevated risk of lung cancer who undergo screening with LDCT compared with either no screening or screening with another modality? 
- What is the rate death or complications resulting from radiation exposure among individuals at elevated risk of lung cancer who undergo screening with LDCT compared with either no screening or screening with another modality?

- What is the rate of surgery for benign disease among individuals at elevated risk of lung cancer who undergo screening with LDCT compared with either no screening or screening with another modality?

- What is the rate of death from lung cancer among individuals at elevated risk of lung cancer who undergo screening with CXR compared with either no screening or screening with another modality?

- What is the rate of death from lung cancer among individuals at elevated risk of lung cancer who undergo screening with sputum analysis compared with either no screening or screening with another modality?

The search and data extraction for the first four questions were conducted as part of a multisociety collaborative effort (American Cancer Society, American College of Chest Physicians [ACCP], American Society of Clinical Oncology, and National Comprehensive Cancer Network [NCCN]). ${ }^{2}$ This multisociety systematic review and guideline explicitly focused on LDCT screening and provided the basis of the LDCT screening recommendations that are part of the third edition of the ACCP Lung Cancer Guidelines. The process, inclusion and exclusion criteria, and methods of data abstraction for these questions have been described elsewhere as has the method of approval for this portion of the guideline. ${ }^{2}$ In this article, we summarize some of the key findings from the multisociety review as well as provide a qualitative assessment and discussion of related issues regarding LDCT screening that extend the earlier publication. ${ }^{2}$

For the latter two questions (regarding CXR and sputum screening) the search was carried out as outlined by Lewis et $\mathrm{a}^{1}$ in "Methodology for Development of Guidelines for Lung Cancer" in the ACCP Lung Cancer Guidelines. ${ }^{1}$ Details of the search strategy are available on request. Only randomized controlled trials (RCTs) reporting a mortality outcome were included, and the search was limited to English language and articles published since 2000 (to correspond to the first edition of the ACCP Lung Cancer Guidelines). New studies or studies with updated mortality data were included and reviewed together with those identified in the first edition of the ACCP Lung Cancer Guidelines.

The CXR and sputum screening recommendations were approved according to the third edition of the ACCP Lung Cancer Guidelines process as described by Lewis et al. ${ }^{1}$ The data for LDCT screening was presented and the recommendations discussed at the ACCP Lung Cancer Guidelines (3rd ed) panel meeting and included panel voting with the understanding that the recommendations had already been approved by the ACCP (and other organizations) through the multisociety guideline development process and represented established policy.

\subsection{BACKGROUND - GENERAL IsSUES RELATED TO SCREENING}

There are several aspects about screening for a disease that make assessment of this intervention different from assessment of treatment of a disease. Screening is applied to an asymptomatic healthy population. Because the majority of screened individuals are likely not to have the disease and would go on to live their lives normally if screening were not done, any potential harms resulting from screening are seen in a different light than potential complications of evaluation or treatment in patients with lung cancer. Furthermore, it is inherent that a relatively large number of people must be screened to find a small number with the disease who might benefit from screening. A situation is thus created where the number of patients benefiting may seem numerically small compared with treatment interventions. Screening also assumes that effective treatment is available (and given) if lung cancer is found; in the absence of effective treatment, screening by definition can never result in a benefit. Thus, the concepts, outcomes, and magnitude of differences are fundamentally different for a screening test than for a treatment intervention. Because the outcomes and issues related to screening are not intuitive and are markedly different from what most clinicians and people in general are used to, it is important that sufficient time is taken by clinicians involved and patients considered for screening to explain and understand the data and the issues. Studies have shown that physicians poorly understand the data and concepts related to a screening intervention. ${ }^{4,5}$

The ultimate goal of a screening test is to reduce the chance of dying of the disease that is being screened for without causing harm in the process. Thus, overall mortality in the population and mortality specifically from lung cancer are the primary outcome measures. The assumption is that screening will find lung cancer at an earlier stage, when more effective treatment is available. The key surrogate measure, therefore, is a reduced number of patients with advanced stage disease (stage IV) at diagnosis because these patients most consistently account for the rate of death from lung cancer. Because the goal of a screening test is to affect the population, outcome measures must be assessed relative to this population (eg, stage distribution of lung cancers in the population). As stated earlier, the rate of death from lung cancer in the population is appropriate, but the survival of only those patients given a diagnosis of lung cancer does not provide an assessment of the impact of screening.

A concept that often is unrecognized is the fact that the process of screening alters the nature of the disease. A more exact wording is that the mixture of patients with the disease is different in a screened vs an unscreened cohort. All diseases, including lung cancer, consist of a spectrum, with some patients having an aggressive form and others a more indolent form of the disease. A very rapidly growing lung cancer (eg, most small cell lung cancers) will advance from an undetectable state to a symptomatic or incurable state during the interval between screening interventions. A very slow growing cancer, on the other hand, exists in a detectable, yet early stage for a prolonged period and, thus, is much more likely to be detected than tumors in an unscreened population. 
This fact underscores the need to look at outcome measures that assess the whole population as well as at the need to focus on randomized trials of screening.

Lung cancers may be so indolent that they never progress to a state of causing symptoms or limiting life. If such a cancer is diagnosed during life, it is called an overdiagnosed cancer. However, there is no way to definitively identify such a tumor except in retrospect. Therefore, the concept of overdiagnosis is useful when assessing outcomes for a screened vs an unscreened population in retrospect. If more cancers are diagnosed in the screened population but lung cancer mortality is unchanged, then by definition, these are overdiagnosed cases (which includes cases for which treatment was ineffective or not administered). However, for a clinician who is trying to predict the prognosis for a patient, the concept of overdiagnosis has little practical application. ${ }^{6}$

A key concept is that screening is a process, not simply a test. It is a complex interplay of selection of a population with sufficient risk (and low enough competing risks), the value of the screening test, the interval between screening tests, the availability of effective treatment, the risk of complications or harms resulting from screening, motivation of the screened individuals, and the rigorousness of compliance with screening and with treatment recommendations. Each factor influences the outcomes of screening and can tip the balance in favor of or against screening. Focusing only on the screening test itself ignores the fact that all the pieces need to work in concert for screening to be effective.

\subsection{RESUlTS}

\subsection{Screening With CXR or Sputum Analysis}

The first edition of the ACCP Lung Cancer Guidelines summarized the data from RCTs using CXR or sputum analysis to screen for lung cancer. ${ }^{7,8}$ The present search yielded one new RCT that assessed the value of screening with CXR or sputum, ${ }^{9}$ three systematic reviews, ${ }^{10-12}$ and two repeat publications with updated data. ${ }^{13,14}$ These were combined with studies identified in the first edition and in the following analysis.

Two studies analyzed the addition of sputum analysis every 4 months to annual CXR vs annual CXR alone..$^{15-17}$ These studies had a matching design and accrued similar patients, which led to a recent combined analysis with updated follow-up. ${ }^{14}$ A nonsignificant trend to reduction in lung cancer mortality was observed with the addition of sputum analysis (rate ratio [RR], 0.88; 95\% CI, 0.74-1.05). Analysis of subgroups revealed that the trend was seen particularly for squamous cell cancer (RR, 0.79; 95\% CI, 0.54-1.14) and especially for patients with higher smoking exposure (RR, 0.81; 95\% CI, 0.67-1.00). ${ }^{14}$ These studies enrolled men aged $>45$ years who had smoked at least one pack per day in the year prior to enrollment (from 1973-1978, with data reported for 9 years of follow-up). Thus, the risk of developing lung cancer was somewhat low, and with a total of 20,426 patients, it is possible that a significant lung cancer mortality reduction might have been missed if applied to a higher risk or larger cohort. Limited data about harms were reported, but two deaths occurred in patients who were found to have benign disease.

Four RCTs compared more frequent CXR screening (every 4 or 6 months) with less frequent screening (CXR every 1-3 years). ${ }^{13,15,18,19-23}$ Some of these studies also involved sputum screening (in both arms). ${ }^{18,19}$ None reported a reduction in lung cancer mortality; in fact, all reported a slight increase in lung cancer deaths in the more frequent screening arms. A metaanalysis showed an RR of 1.11 (95\% CI, 0.95-1.31). ${ }^{11}$ The same results were reported in a Cochrane metaanalysis, which concluded that there was no evidence to support screening with CXR. ${ }^{12}$ The patients were primarily men, with starting ages between 35 and 45 years, and were limited to smokers in two studies. The total number of patients involved was 81,303. One study $(\mathrm{n}=55,034)$ enrolled patients between 1960 and 1964; the others enrolled patients during the 1970s. ${ }^{24}$ Furthermore, the compliance in these studies was generally only about $60 \%$. Of note, these studies were not designed to assess screening vs no screening because they did not have a true observationonly arm. 7,8

Since 2002, only one additional RCT has been conducted (the Prostate, Lung, Colorectal and Ovarian trial $[\mathrm{PLCO}])$, and the results with respect to CXR screening for lung cancer were recently reported. ${ }^{9}$ From 1993 to 2001, 154,901 participants aged 55 to 74 years were randomized at 10 sites to a posteroanterior CXR at baseline followed by an annual CXR for 3 more years vs usual care. The participants were followed until either December 2009 or a maximum of 13 years. Ten percent were current smokers, $42 \%$ former smokers, and $45 \%$ never smokers; $11 \%$ had a first-degree relative with lung cancer. Compliance with screening was fair (decreasing from $87 \%$ at baseline to $79 \%$ at year 3 ) and was worst in those at highest risk $(87 \%$ overall in never smokers vs $79 \%$ in current smokers). ${ }^{25}$ In contrast to the earlier studies, the contamination rate in the control arm was relatively low (11\% had a CXR).

The cumulative lung cancer mortality was the same in both arms (RR, 0.99; 95\% CI, 0.87-1.22; $P=.48)$ (Fig 1). This was not affected by the smoking status, with similar RRs for never, former, and current smokers. Of all participants, $21 \%$ matched the entry criteria 
FiguRE 1. [Section 3.1] Rate ratios of lung cancer mortality. Lung cancer mortality rate ratios for chest radiograph-screened vs usual care participants in the PLCO.$^{9}$ NLST $=$ National Lung Screening Trial; PLCO $=$ Prostate, Lung, Colorectal and Ovarian trial.

\section{PLCO: Lung Cancer Mortality}

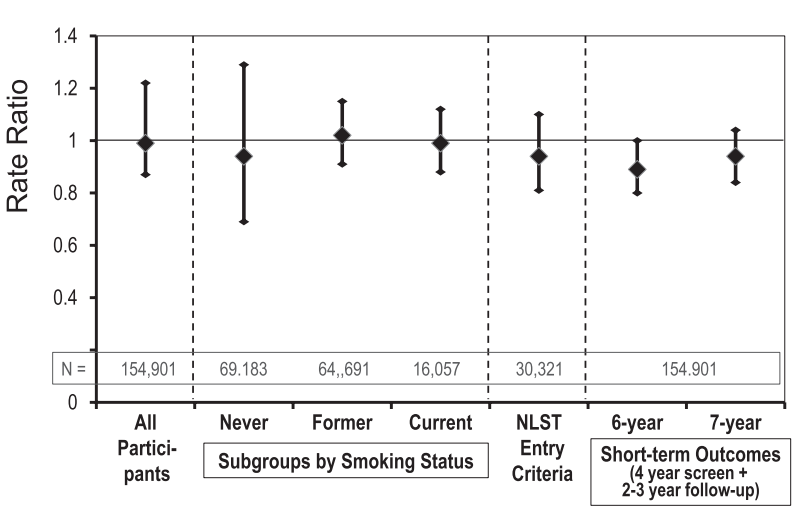

of the National Lung Screening Trial (NLST); there was no difference in lung cancer mortality for this subset either.

Although the incidence of cancer varied significantly by smoking history, the RR between the screened and control arms did not (incidence per 10,000 person-years, $3.1,23$, and 83 ; RR, 1.06, 1.12, and 1.00 for never, former, and current smokers, respectively). Cumulative lung cancer incidence was slightly, but not significantly higher in the screened arm (20.1 vs 19.2 per 10,000 person-years; RR, 1.05; 95\% CI, 0.98-1.12). The absolute number of stage III and IV non-small cell lung cancers (NSCLCs) was the same, although there were more stage I cancers in the screened vs usual care arm (462 vs 374). There was no difference in the histologic subtypes of cancer between arms. The treatment given for NSCLC was also similar.

The long follow-up in the PLCO is both a strength and a weakness. Only $18 \%$ of screening arm cancers were detected $(12 \%$ detected between screening rounds [interval cancers], $11 \%$ in nonadherent participants, and 59\% during follow-up [after screening]), potentially diluting the screening effect. However, no mortality difference was apparent when the results were analyzed after only 6 to 7 years $(4$ years of screening and 2-3 years of follow-up to detect potentially missed cancers in the usual care cohort).

In summary, a large, appropriately designed trial found no benefit to annual CXR screening vs no screening. Potential limitations caused by inclusion of lowrisk participants (never smokers) or long follow-up are countered by similar results in subgroup analyses (still involving large cohorts). Although because of design differences the PLCO is not directly comparable with the earlier studies, taken together, these studies demonstrate clearly that there is no benefit to screening for lung cancer using CXR. No clear documentation of harms resulting from screening is available from these studies.

\subsection{Recommendations}

3.2.1. In patients at risk for developing lung cancer, screening for lung cancer with CXR once or at regular intervals is not recommended (Grade 1A).

Remark: These results should not be interpreted as diminishing the role of CXR in evaluating patients with pulmonary symptoms (an entirely different situation than screening asymptomatic individuals).

3.2.2. In patients at risk for developing lung
cancer, screening for lung cancer with sputum
cytology at regular intervals is not suggested (Grade 2B).

\subsection{Screening With LDCT}

A systematic review ${ }^{2}$ of LDCT screening for lung cancer from 1996 to 2011 identified eight RCTs ${ }^{26-33}$ and 13 prospective cohort studies ${ }^{34-46}$ that met the inclusion criteria. These studies focused on middleaged or older individuals with a history of smoking; however, the age criteria and extent of smoking exposure varied. It is difficult to assess the average risk of developing lung cancer in the cohorts included in the different studies; for those meeting the minimum criteria, the estimated risk of developing lung cancer within the next 10 years varied from $2 \%$ in some studies to about $0.5 \%$ in others.

Results mature enough to evaluate the effect of LDCT screening on the rate of death from lung cancer in the screened population is available from only three RCTs. ${ }^{26,30,33}$ The NLST found a dramatic $20 \%$ reduction in lung cancer mortality in participants screened by LDCT scan compared with those screened with CXR (RR, 0.80; 95\% CI, 0.73-0.93; $P=.004) .{ }^{26}$ No difference in lung cancer mortality was reported in the Detection and Screening of Early Lung Cancer by Novel Imaging Technology and Molecular Essays Trial (DANTE) (RR, 0.97; 95\% CI, $0.71-1.32 ; P=.83)^{30}$ or the Danish Lung Cancer Screening Trial (DLCST) (RR, 1.15; 95\% CI, 0.83-1.61; $P=.06) .{ }^{33}$ The NLST is by far the larger study $(53,454$ vs 2,811 and 4,104 participants), involved a longer follow-up (median, 6.5 vs 2.8 and 4.8 years), and included participants at slightly higher risk (30 vs 20 and 20 pack-years of smoking) compared with DANTE and DLCST, respectively. The NLST involved three annual rounds of screening vs five in DANTE and DLCST, with the comparison being with CXR in NLST and with observation alone in DANTE and 
DLCST. Similar high compliance (95\%, 91\%, and 96\%) and low contamination rates $(4.3 \%, 6.1 \%$, and $2.8 \%)$ were reported in the NLST, DANTE, and DLCST, respectively. ${ }^{30,47,48}$ Taken together, the data support a significant reduction in deaths from lung cancer (291 vs 474; OR, 0.82; 95\% CI, 0.72-0.94; $P=0004$ ) resulting from several rounds of LDCT screening of individuals at risk. ${ }^{2}$

DANTE and DLCST were too small to expect to show any statistical significance; however, the fact that there was no trend similar to NLST toward fewer deaths from lung cancer is surprising. This is corroborated by finding no decrease in the rate of higher stage cancers in DANTE and DLCST, whereas there clearly was in NLST (17 vs 17 patients in DANTE, nine vs nine in DLCST, and 348 vs 457 in NLST had stage IIIB-IV NSCLC in the LDCT imaging vs control arms, respectively). The differing outcomes in DANTE and DLCST could be due to chance alone, to shorter follow-up, or to a lower inherent risk among the screened population. These results suggest that we should be cautious in assuming that the other RCTs of LDCT screening will show the same results as the NLST. Together, the completed, but not yet mature additional RCTs of LDCT screening involve about 25,000 participants (nearly one-half the size of the NLST); it will take until approximately 2015 before most of these additional data are available.

A concern that often is raised is the high rate of finding an abnormality on an LDCT scan that is not, in fact, cancer. Across studies, the average nodule detection rate was around $20 \%$ (Fig 2) but varied from $3 \%$ to $30 \%$ in RCTs and $5 \%$ to $51 \%$ in cohort studies. In general, there is a trend toward lower nodule detection rates in repeat screening rounds, but the data and reporting are inconsistent. Most studies reported that $>90 \%$ of nodules were benign. Perhaps the best way to assess the potential negative impact of this issue is the rate of invasive procedures that were performed and how often this was undesirable, meaning performed for benign disease. In the organized, controlled setting of the LDCT randomized and cohort screening studies (almost all of which involved major academic medical centers), the rate of invasive procedures was low, although it varied from $1 \%$ to $4 \%$ (Fig 3). However, about $25 \%$ of invasive procedures were done in patients with what was eventually shown to be benign disease (range, 0\%-45\%) (Fig 3).

FIGURE 2. [Section 3.3] Frequency of screening participants with a nodule detected on baseline LDCT scan and percentage of nodules eventually proven to be benign in LDCT studies. A, Percentage of all participants screened with LDCT imaging who had a nodule detected at baseline screening. B, Percentage of patients with a lesion identified at baseline LDCT screening that was eventually found to be benign. Cohort = single-arm cohort studies of LDCT; DANTE = Detection and Screening of Early Lung Cancer by Novel Imaging Technology and Molecular Essays Trial; DLST $=$ Danish Lung Cancer Screening Trial; LDCT = low-dose CT; LSS = Lung Screening Study; NELSON = Dutch Belgian Randomised Lung Cancer Screening Trial; RCT $=$ randomized controlled trial. See Figure 1 legend for expansion of other abbreviation.

A
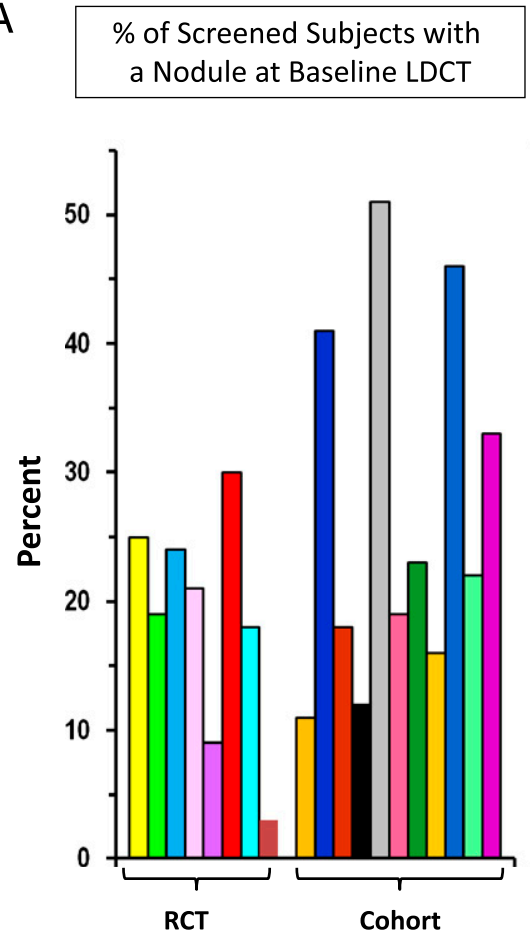

B

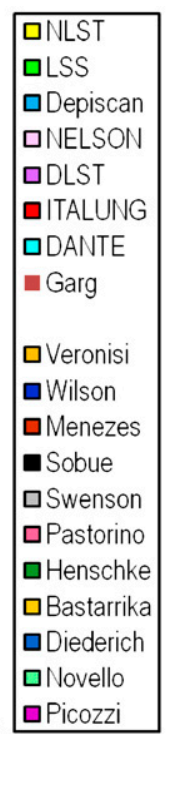

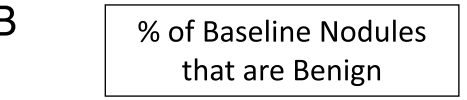

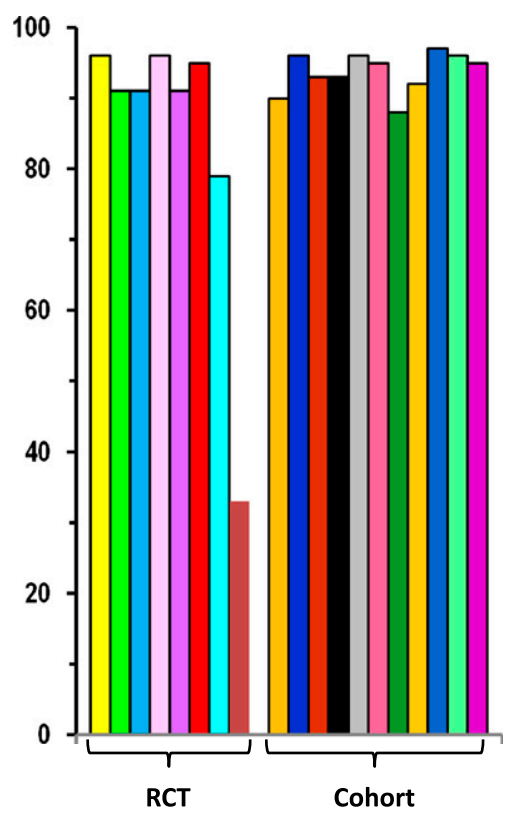


FiguRE 3. [Sections 3.3, 4.2] Frequency of patients undergoing a surgical biopsy or procedure and percentage of such surgical biopsies or procedures done for a benign lesion in LDCT studies. A, Percentage of all participants screened with LDCT imaging who underwent a surgical biopsy or procedure to evaluate a detected nodule at baseline screening. B, Percentage of patients who underwent a surgical biopsy or procedure for a lesion identified at baseline LDCT screening that was found to be benign. * Both surgical and nonsurgical (ie, needle aspiration) biopsies were reported together. See Figure 1 and 2 legends for expansion of abbreviations.

A
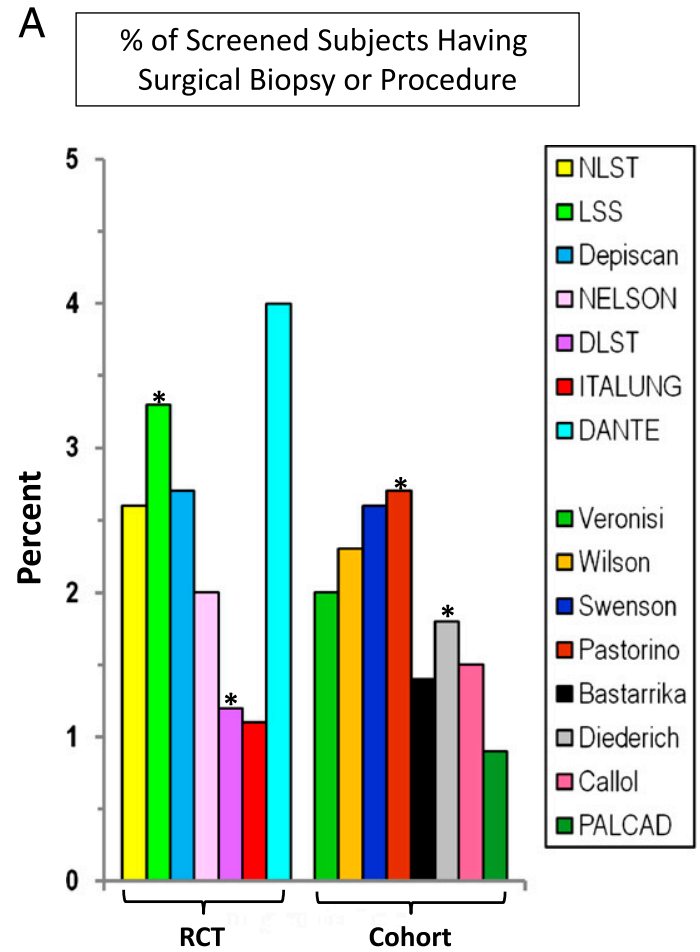

B

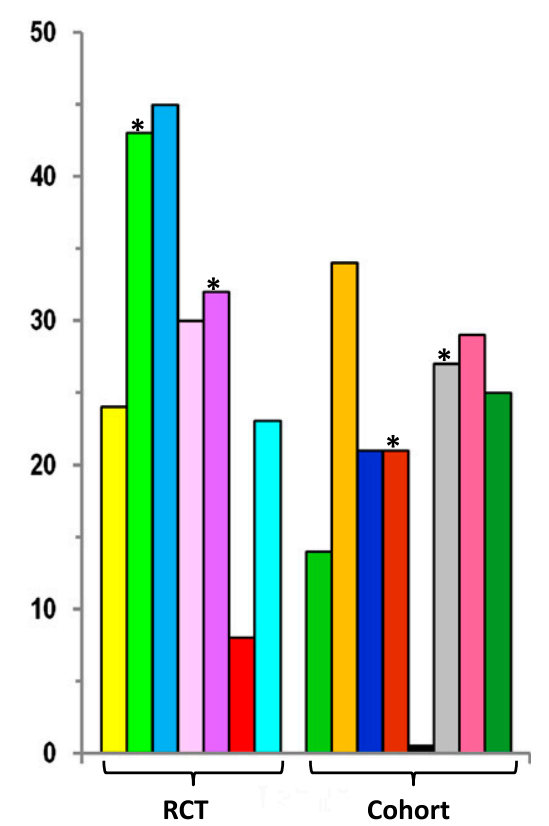

The most serious concern is the risk of death or major complications as a result of screening. Of course, this must be compared with the rate in nonscreened patients and recognize that some deaths may be unrelated events that happened to occur after a screening test. Limited data are available that carefully assesses this concern; the NLST provides the best source of such data at this time. Overall, the frequency of deaths in NLST that occurred within 2 months of a diagnostic evaluation of a detected finding was eight per 10,000 individuals screened by LDCT scan vs five per 10,000 individuals screened by CXR. Some deaths were presumably unrelated, as 1.9 and 1.5 per 10,000 occurred within 2 months when the diagnostic evaluation involved only an imaging study. Focusing only on patients who had detected nodules eventually found to be benign, the risk of death or major complications following diagnostic events (including imaging) was 4.1 and 4.5 in the LDCT screening arm vs 1.1 and 1.5 per 10,000 participants in the CXR arm. In summary, there was an appreciable increase in the rate of death or major complications resulting from investigation of screening findings from LDCT imaging and specifically in individuals who had only benign lesions. Nevertheless, in

the highly organized NLST setting, the rate of such events was quite low.

Another concern is the amount of radiation. An appropriately performed LDCT scan (about $1.5 \mathrm{mSv}$ ) is less than the average annual background radiation received in the United States (about 3-4 mSv). However, nodules requiring further imaging rapidly drive up the dose these patients receive. The average dose received per participant over 3 years in the NLST is estimated at $8 \mathrm{mSv}$. Another study found an average exposure of 6.2 to $6.8 \mathrm{mSv}$, with a maximum of 20 to $22 \mathrm{mSv}$ over 4 years. ${ }^{49}$ For comparison, an average of $20 \mathrm{mSv}$ per year and a maximum in any given year of $50 \mathrm{mSv}$ is the limit allowed for at-risk workers. The American Association of Physicists in Medicine recently stated, "Risks of medical imaging at effective doses below $50 \mathrm{mSv}$ for single procedures or $100 \mathrm{mSv}$ for multiple procedures over short time periods are too low to be detectable, and may be nonexistent."50 From estimates of several official bodies and commissioned studies, ${ }^{51,52}$ which are based on dose extrapolations from atomic bombings and many studies of medical imaging, ${ }^{53,54}$ we estimate that the risk of a radiation-induced cancer in the NLST is approximately one cancer death in 2,500 screened participants. ${ }^{2}$ 
Therefore, the benefit in preventing lung cancer deaths in NLST is considerably greater than the radiation risk (at least in older individuals given that radiationinduced cancer typically develops $10-20$ years later).

\subsection{Recommendations}

3.4.1. For smokers and former smokers who are age 55 to 74 and who have smoked for 30 pack-years or more and either continue to smoke or have quit within the past 15 years, we suggest that annual screening with LDCT should be offered over both annual screening with CXR or no screening, but only in settings that can deliver the comprehensive care provided to NLST participants (Grade $2 \mathrm{~B}$ ).

Remark: Counseling should include a complete description of potential benefits and harms, so the individual can decide whether to undergo LDCT screening.

Remark: Screening should be conducted in a center similar to those where the NLST was conducted, with multidisciplinary coordinated care and a comprehensive process for screening, image interpretation, management of findings, and evaluation and treatment of potential cancers.

Remark: A number of important questions about screening could be addressed if individuals who are screened for lung cancer are entered into a registry that captures data on follow-up testing, radiation exposure, patient experience, and smoking behavior.

Remark: Quality metrics should be developed such as those in use for mammography screening, which could help enhance the benefits and minimize the harm for individuals who undergo screening.

Remark: Screening for lung cancer is not a substitute for stopping smoking. The most important thing patients can do to prevent lung cancer is not smoke.

Remark: The most effective duration or frequency of screening is not known.

3.4.2. For individuals who have accumulated fewer than 30 pack-years of smoking or are either younger than age 55 or older than 74 , or individuals who quit smoking more than 15 years ago, and for individuals with severe comorbidities that would preclude potentially curative treatment and/or limit life expectancy, we suggest that CT screening should not be performed (Grade 2C).

*Recommendations 3.4 .1 and 3.4 .2 were approved through a previous multisociety guideline development process and published elsewhere ${ }^{2}$ and are included here for completeness. The majority of panel members at the ACCP Lung Cancer Guidelines (3rd ed) meeting voted in agreement with both recommendation 3.4.1 (>80\% of panelists) and 3.4.2 (>50\% of panelists); ACCP Lung Cancer Guidelines (3rd ed) recommendations require $67 \%$ approval as described more fully in the methodology article. ${ }^{1}$

\subsection{Discussion of Issues Regarding SCREENING FOR LUNG CANCER}

\subsection{Participant Selection}

At present, the only population for which there is a demonstrated benefit is that defined by the NLST: smokers with at least 30 pack-years of exposure, who are aged 55 to 74 years, and who quit $<15$ years ago. It is important to note that there is demonstration of benefit only for the entire NLST population; the risk of developing lung cancer ranges from about $2 \%$ to $>20 \%$ over 10 years for individuals at the low or high end of the NLST inclusion criteria, as estimated from risk prediction models. ${ }^{55,56}$ Estimating an average risk for the entire NLST population is difficult from the data reported, but can be estimated to be roughly $10 \%$ over 10 years.

Expanding screening to cohorts other than those included in the NLST is probably not warranted at this time unless it is in the context of a research study. In fact, we do not know that those at the lower end of inclusion in the NLST benefit, much less if screening is extended to even younger participants or to those with less smoking exposure. The fact that DANTE and DLCST, which included patients at lower risk, do not appear to have a similar benefit should engender caution. ${ }^{30,33}$ Furthermore, expansion to older age groups must take into account an increasing risk of competing causes of death, yet models to estimate this are limited. ${ }^{57,58}$ Maturation of the results of ongoing RCTs of lung cancer screening and examination of details of the NLST participants may provide further insight. Because additional RCTs in other cohorts are unlikely to be conducted, careful modeling studies are needed; several are currently under way. However, until such data are available, expansion of screening to cohorts beyond the NLST outside a research study must be viewed as speculative and not evidence based.

The NCCN has come forth to recommend screening for individuals as young as 50 years with $\geq 20$ pack-years exposure (apparently regardless of how long after smoking cessation) and one of the following additional risk factors: radon exposure, family history, pulmonary disease history, occupational exposure, or other cancers. ${ }^{59}$ Although these are recognized as independent 
risk factors for lung cancers, most have not been incorporated into a risk prediction model. Therefore, no assessment can be made at this time with regard to the impact of these factors or how high the risk of lung cancer is in the expanded NCCN cohort beyond that of NLST. The NCCN recommendations primarily represent a consensus among the experts at the NCCN centers, as is reflected in the NCCN grading system. Because the ACCP process requires scientific evidence before making a guideline recommendation, the ACCP recommends screening only for the cohort defined by the NLST at this time. The ACCP suggests that screening outside a research study for additional cohorts should not be done until further data are available.

The NLST population had a much higher level of education than the average smoking population in the United States ${ }^{60}$ and may have affected compliance with both screening and follow-up as well as competing causes of death. Even for participants meeting NLST criteria, a better understanding of how implementation in a broader context would actually work out is needed.

\subsection{Minimizing Harms}

The potential to achieve a $20 \%$ reduction in deaths from the cancer that accounts for almost one-third of cancer deaths is a tremendous step forward and may well represent the largest impact on cancer deaths resulting from a single intervention in several decades. Concerns have been raised about potential harms from lung cancer screening, mainly in terms of potential complications from unnecessary procedures done to investigate what are found to be benign, inconsequential nodules. ${ }^{61}$ However, the data from the NLST indicate that in the setting in which NLST was conducted, the chance of major harms was very low; the risk of death or major complications following diagnostic events (including imaging) for what turns out to be a benign nodule is 4.1 and 4.5 per 10,000 . Furthermore, about one-half of these events occurred when the diagnostic event was only an imaging study, suggesting that in some of these cases, the morbidity and mortality may have been unrelated to screening. ${ }^{2}$

Nevertheless, there is reason to be concerned about an increase in harms as CT screening is implemented more broadly. The NLST setting was highly structured, with extensive quality control, and involved sophisticated tertiary medical centers. The same is true for the other RCT studies and cohort studies of LDCT screening for lung cancer. ${ }^{2}$ Nevertheless, even in this setting, there is large variability in the percentage of patients who underwent a surgical procedure, and the percentage of patients undergoing a surgical procedure who ended up having a benign nodule varied from $<10 \%$ to $>40 \%$ (Fig 3). ${ }^{2}$ More concerning are US hospital statistics in different regions that show great variation in the rate of invasive procedures for pulmonary nodules and nearly twofold differences in the rate of complications from these procedures. ${ }^{62}$ The fact that $1.5 \%$ to $4 \%$ of patients experiencing such complications died during that hospitalization underscores that we must be careful about potential harms beyond that seen in the NLST if screening is implemented more broadly.

An issue is the fact that LDCT screening detects many nodules (varying from 3\%-42\%). Given the fear of lung cancer that leads people to undergo LDCT screening, there is pressure to investigate these nodules further, even though the majority (around 97\%) are benign, and suggests that preemptive counseling is important to avoid unwarranted concern. It also underscores the need for a formal process to be in place to evaluate these lesions thoughtfully. Biopsy of many benign lesions is not appropriate, but lack of action for a growing malignant lesion is also undesirable. The centers at which LDCT screening studies have been conducted (including in the NLST) have had such processes in place. Predetermined algorithms can minimize the number of further imaging studies or invasive biopsies to what is truly necessary. The cost of the process of screening should be reimbursed fairly; establishing a discounted rate as a strategy to capture patients with the rationale that a large number of subsequent procedures and interventions will make up for the initial loss creates a structure that can increase harms from excessive investigation of benign nodules. Furthermore, those who offer only an LDCT scan without being part of a structured multidisciplinary program reflect a poor understanding of the totality of the process of CT screening.

Potential harms from surgical procedures, especially for benign nodules, are a particularly prominent issue. Nevertheless, although data are only available from a few studies, $26,63,64$ they show that such risks are quite low, and, in general, appear to predominantly involve risks from major resection of actual lung cancers. ${ }^{26} \mathrm{In}$ the NLST, the mortality from such resections was $1 \%,{ }^{26}$ which compares favorably to that reported from specialized thoracic centers. ${ }^{65,66}$ For comparison, in general US databases, mortality of major pulmonary resections is $3 \%$ to $5 \% .{ }^{67-70}$ This rate is consistent with the observation that the majority of centers involved in both the RCT and the prospective controlled CT screening studies had specialized thoracic surgical services, and many had thoracic surgical training programs. ${ }^{2}$

It is well established that video-assisted thoracic surgery (VATS) results in less postoperative morbidity. $69,71-73$ On average, about $25 \%$ of the thoracic surgical procedures were done for benign nodules (Fig 3 ). It is much 
easier to accept this rate if the procedure is a diagnostic VATS resection rather than a thoracotomy. Because the penetration of major VATS skills is much less among cardiac and general surgeons and outside specialized centers, ${ }^{67,68}$ several organizations, including the ACCP panel, recommend that a good quality screening program include the availability of major VATS (Fig 4).

Various major societies and organizations active in this arena have issued formal statements outlining components of an appropriate lung cancer screening program. These are summarized in Figure 4. There is consistency among these recommendations, with the differences being the number of aspects that were addressed. One frequent recommendation calls for the development and implementation of quality metrics to assess whether the screening processes are actually achieving the desired balance of benefits and harms. Such metrics are not yet available.

\subsection{Implementation}

Data from one trial show that LDCT screening in a highly structured setting with tightly controlled selection can lead to a decrease in the rate of death from lung cancer. How well these results can be achieved if screening is implemented on a broader scale is unclear. If the process is duplicated, it is reasonable to assume that this will be the case; however, the variability in nodule detection rates and in rates of interventions in different studies and regions in the United States in general raises concern that the results may differ if screening is implemented in a less structured manner. Several of the major organizations that have developed position statements on CT screening have called for implementation of demonstration projects to assess how well LDCT screening can be implemented on a broader basis (Fig 4). How important various organizational and structural aspects of LDCT screening programs are is unclear and requires study. Furthermore, the impact of the components of a CT screening program as recommended by the major societies is unclear. Such questions could be addressed in demonstration projects and would be important in the development of validated quality metrics.

An issue with the implementation of CT screening is appropriate selection of individuals to be screened. In the setting of a formal study, and in the absence of clear data regarding a benefit, criteria can be set and strictly adhered to. Given data for a benefit for some, it is likely to be much more difficult to adhere to strict criteria with broader implementation, especially if risk factors not included in the NLST are included. Some risk prediction models have been developed, and some have been validated in certain settings, $55,74-80$ but applicability on a broad scale needs to be demonstrated. Systems that take into account competing causes of death also need to be developed. ${ }^{57,58}$ Until such tools are well established, adherence to strict selection criteria will be challenging. An important aspect is the ability to reassure individuals who are at low risk and not appropriate for screening that they are, in fact, at low risk instead of leaving them with

FIGURE 4. [Sections 4.2, 4.3] Components of a CT scan screening program as proposed by major organizations.

\begin{tabular}{|c|c|c|c|c|c|c|c|}
\hline Components Recommended & $\begin{array}{c}\text { ACCP } \\
\text { LC III } \\
\text { article }^{83}\end{array}$ & $\begin{array}{c}\text { Multi- } \\
\text { society }^{\mathrm{a}} \\
\text { guideline }^{2}\end{array}$ & $\mathrm{ACS}^{84}$ & IASLC $^{85}$ & AATS $^{86}$ & $\mathrm{NCCN}^{59}$ & STS $^{87}$ \\
\hline Multispecialty program & Yes & Yes & Yes & Yes & Yes & Yes & Yes \\
\hline Careful participant selection & Yes & Yes & Yes & Yes & Yes & Yes & Yes \\
\hline Participant Education/Counseling & Yes & Yes & Yes & - & - & Yes & Yes \\
\hline Smoking cessation & Yes & Yes & Yes & Yes & Yes & Yes & Yes \\
\hline CT with quality controls & Yes & Yes & Yes & Yes & Yes & Yes & Yes \\
\hline Defined process for scan interpretation & Yes & Yes & Yes & Yes & - & - & Yes \\
\hline Defined intervention algorithm & Yes & Yes & Yes & Yes & Yes & Yes & Yes \\
\hline Quality metrics & Yes & Yes & - & Yes & - & - & Yes \\
\hline Registry/data collection & Yes & Yes & - & Yes & Yes & - & Yes \\
\hline Ongoing research participation & Yes & Yes & - & Yes & Yes & - & Yes \\
\hline Video-Assisted Thoracic Surgery & Yes & - & - & Yes & Yes & - & Yes \\
\hline Demonstration Project needed & Yes & Yes & - & Yes & - & - & - \\
\hline
\end{tabular}

AATS = American Association of Thoracic Surgery; ACCP = American College of Chest Physicians; ACS = American Cancer Society; ASCO = American Society of Clinical Oncologists; ATS = American Thoracic Society; IASLC = International Association for the Study of Lung Cancer; LC III = Lung Cancer Guidelines (3rd ed); NCCN = National Comprehensive Cancer Network.

aCCP, ASCO, and ATS 
the perception of being denied access to a screening program because of arbitrary rules.

The ability to reach the population who is most likely to benefit from screening is an issue. Data have shown that those with high-risk lifestyles are less interested in screening and even in treatment. ${ }^{81,82}$ Despite the smoking history requirements, the NLST population was not completely representative of the US population at elevated risk due to smoking exposure. ${ }^{60}$ Whether a large proportion of those at risk can be reached through educational programs is unclear. Nevertheless, even if the maximal benefit of CT screening is not achieved because certain at-risk cohorts cannot be reached or because screening is less effective, this would not alter the role in a cohort similar to the NLST.

Another issue with implementation is whether primary-care physicians are able to assume a major role in the selection and management of patients for lung cancer screening. For breast, cervical, and prostate cancer screening, definition of the target populations and the screening tests are simpler, as are further investigations of screen-detected abnormalities. The biggest difference may be that general education, systems of care, and quality metrics are well established for breast, cervical, and colorectal screening. However, despite this, the level of understanding of statistics related to screening among primary-care physicians often is limited ${ }^{4,5}$ and may be a particular issue for a new screening test as complex as LDCT imaging for lung cancer. The fact that lung cancer is so clearly linked to a modifiable risk factor (ie, smoking) makes the discussion inherently different from that for breast or colon cancer screening. If primary-care physicians are asked to play the primary role in counseling patients about whether they should be screened as well as in the interpretation of results, a major educational effort is needed. How effectively this can be accomplished would have a major impact on how and how well LDCT screening can be broadly implemented. It may be more feasible to establish an assessment and counseling component staffed by individuals with special lung cancer or screening expertise as the portal of entry into an LDCT screening program.

\subsection{Lingering Questions}

There are many unanswered questions at this point. A major question is whether other LDCT screening trials will demonstrate a reduction in lung cancer mortality. It is unusual to base a major health-care policy change on the results of one study. This is countered to an extent by the fact that the NLST was such a large, well-done study. Nevertheless, the fact that DANTE and DLCST do not mirror the NLST results suggests that we at least keep an open mind as further results become available over the coming years.

Another major question is what will be the results of ongoing screening beyond 3 years. Data from studies underway will help, and modeling studies are needed. Furthermore, studies of the implementation of CT screening are needed to see whether assumptions made from existing data in building models are consistent with what is actually done (ie, participant selection, number and dose of CT scans, compliance). The process of evaluation of screen-detected lesions plays a central role in balancing benefits and harms, and this will likely undergo refinement. Ongoing study and adjustment will be necessary as the process evolves.

Other lingering questions include how well LDCT screening can be implemented outside a tightly controlled research setting, as discussed previously. Demonstration projects are the ideal way to evaluate this question. Another issue is to what extent the selection should be extended beyond the NLST criteria. As discussed in a previous section, the results of additional LDCT screening studies and modeling studies are needed. Finally, we have little information about the appearance of lesions that were selected for biopsy or resection in the CT screening studies.

The multitude of additional questions creates a compelling argument that the implementation of CT screening should include a registry; the relatively low event rate for many of the outcomes argues that this should be done as a mandatory, more or less uniform component of screening programs. Funding such an aspect of implementation of LDCT screening is a potential problem, but it is likely to result in cost savings by allowing the process of CT screening for lung cancer to be done in a way that becomes increasingly more effective. Furthermore, a registry would facilitate the tracking of quality metrics; avoidance of poor quality in screening programs should also reduce costs for the health-care system.

\subsection{Balancing Benefits vs Harms}

How one views benefits vs downsides depends on one's perspective and values. A societal view may place greater emphasis on the costs and the ability to carry out other health-care interventions and be in conflict with the view of people who carry an elevated risk of lung cancer. Furthermore, in this as in other population health interventions, the harms and benefits accrue to different people. A consensus of how to balance different viewpoints is needed.

How individuals view the risk of cancer and risk of radiation or invasive interventions can also vary. It is important that these individual decisions are made 
rationally and not out of fear. Fear should be dealt with through information and reassurance rather than through screening. How best to structure a screening program to accomplish this is not clear. We suggest that a good program offers risk assessment and counseling so that people for whom screening appears not to be appropriate are provided the understanding and reassurance to be comfortable. These discussions are likely to be time consuming and require the participation of individuals with particular knowledge of the issues related to LDCT screening for lung cancer.

Assessing the balance of benefits and harms is difficult. The NLST demonstrates a clear benefit. However, the degree of benefit is undoubtedly associated with the underlying risk of developing lung cancer. Although we have some indications (ie, NLST criteria), details about how to apply this to individuals are lacking. We need to understand why DANTE and DLCST results differ from those of the NLST. The procedure-related harms of LDCT screening in the controlled studies appear to be low, but the data are limited. Estimating other risks (eg, psychologic, smoking rate, radiation) is even more difficult. It is likely that there is a cohort that will benefit and other cohorts that will not, but it is difficult at present to assess the balance for the overall population (as defined by NLST criteria, DANTE, or DLCST or by extended criteria). Furthermore, how broader implementation and prolonged screening will affect benefits and risks is difficult to assess at this time.

Taken together, these considerations lead us to suggest screening for patients meeting the NLST criteria, when done in a manner similar to the NLST, but with a level 2 rating because of uncertainty regarding the impact of implementation on how clearly and in what circumstances benefits outweigh risks. The panel anticipates that this may well change with further data, and this guideline will be updated as enough data accrue to alter the recommendations or grading thereof.

\subsection{CONCLUSION}

Lung cancer is by far the leading cause of cancer deaths mainly because of the high proportion of lung cancers diagnosed in an advanced stage. CXR and sputum analysis have not been of benefit as screening tools for lung cancer. However, there is a great deal of excitement and enthusiasm about LDCT screening, and emerging data from maturing studies show promise for a substantial reduction in advanced stage cancers and, thereby, the reduced number of people dying of lung cancer. A systematic review of the evidence available, however, also discloses areas of uncertainty and unanswered questions.
LDCT screening should be suggested to a specific cohort of patients in the context of an organized, comprehensive, multidisciplinary program, including a registry to address lingering questions and to track quality metrics (which need to be established). Screening for lung cancer involves a complex interplay of patient selection, counseling, risk modification, an organized process for screening and scan interpretation, and judgment to minimize further testing to only those instances in which it is necessary. The time and infrastructure necessary to carry out such a process must be recognized and supported. With attention to detail and ongoing refinement of the process, LDCT screening for lung cancer has the potential to have a major impact on the reduction of cancer deaths.

\section{ACKNOWLEDGMENTS}

Author contributions: Dr Detterbeck had full access to all of the data in the study and takes responsibility for the integrity of the data and the accuracy of the data analysis.

Dr Detterbeck: contributed to planning the overall process for development of the article, data analysis, formulation of recommendations, writing, and oversight of the revision of the manuscript during its development.

Dr Naidich: contributed to the review and revisions of the manuscript and recommendations.

Dr Mazzone: contributed to the review and revisions of the manuscript and recommendations.

Dr Bach: contributed to planning the overall process for development of the article, data analysis, formulation of recommendations, and review and revision of the manuscript during its development.

Financial/nonfinancial disclosures: The authors have reported to CHEST the following conflicts of interest: Dr Detterbeck is a member of the International Association for the Study of Lung Cancer International Staging Committee and a speaker in an educational program regarding lung cancer stage classification; both activities are funded by Lilly Oncology (Lilly USA, LLC). He has participated on a scientific advisory panel for Oncimmune (USA) LLC; an external grant administration board for Pfizer, Inc; a multicenter study of a device for Medela; and formerly a multicenter study of a device for Deep Breeze. Compensation for these activities is paid directly to Yale University. Dr Mazzone has participated in industry advisory committee meetings for Oncimmune (USA) LLC and Boehringer Ingleheim Pharmaceuticals, Inc. He also received or will soon receive research support from Metabolomx, Integrative Diagnostics Inc, the National Cancer Institute, and the Ohio Department of Development. Drs Naidich and Bach have reported that no potential conflicts of interest exist with any companies/organizations whose products or services may be discussed in this article.

Role of Sponsors: The American College of Chest Physicians was solely responsible for the development of these guidelines. The remaining supporters played no role in the development process. External supporting organizations cannot recommend panelists or topics, nor are they allowed prepublication access to the manuscripts and recommendations. Further details on the Conflict of Interest Policy are available online at http:// chestnet.org.

Endorsements: This guideline is endorsed by the European Society of Thoracic Surgeons, Oncology Nursing Society, American Association for Bronchology and Interventional Pulmonology, and the Society of Thoracic Surgeons.

Additional information: The supplement table can be found in the "Supplemental Materials" area of the online article. 


\section{REFERENCES}

1. Lewis SZ, Diekemper R, Addrizzo-Harris DJ. Methodology for development of guidelines for lung cancer: diagnosis and management of lung cancer, 3rd ed: American College of Chest Physicians evidence-based clinical practice guidelines. Chest. 2013;143(5)(suppl):41S-50S.

2. Bach P, Mirkin J, Oliver T, et al. Benefits and harms of CT screening for lung cancer: a systematic review. JAMA. 2012;307(22):2418-2429.

3. Siegel R, Naishadham D, Jemal A. Cancer statistics, 2012. CA Cancer J Clin. 2012;62(1):10-29.

4. Wegwarth O, Schwartz LM, Woloshin S, Gaissmaier W, Gigerenzer G. Do physicians understand cancer screening statistics? A national survey of primary care physicians in the United States. Ann Intern Med. 2012;156(5):340-349.

5. Bramwell R, West H, Salmon P. Health professionals' and service users' interpretation of screening test results: experimental study. BMJ. 2006;333(7562):284-286.

6. Detterbeck FC. Cancer, concepts, cohorts and complexity: avoiding oversimplification of overdiagnosis. Thorax. 2012; 67(9):842-845.

7. Bach PB, Kelley MJ, Tate RC, McCrory DC. Screening for lung cancer: a review of the current literature. Chest. 2003; 123(suppl 1):72S-82S.

8. Bach PB, Niewoehner DE, Black WC; American College of Chest Physicians. Screening for lung cancer: the guidelines. Chest. 2003;123(suppl 1):83S-88S.

9. Oken MM, Hocking WG, Kvale PA, et al; PLCO Project Team. Screening by chest radiograph and lung cancer mortality: the Prostate, Lung, Colorectal, and Ovarian (PLCO) randomized trial. JAMA. 2011;306(17):1865-1873.

10. Hunt I, Siva M, Southon R, Treasure T. Does lung cancer screening with chest x-ray improve disease-free survival? Interact Cardiovasc Thorac Surg. 2006;5(4):483-487.

11. Manser RL, Irving LB, Byrnes G, Abramson MJ, Stone CA, Campbell DA. Screening for lung cancer: a systematic review and meta-analysis of controlled trials. Thorax. 2003;58(9): 784-789.

12. Manser R, Irving L, Stone C, Byrnes G, Abramson M, Campbell D. Screening for lung cancer. Cochrane Database Syst Rev. 2004;(1):CD001991.

13. Marcus PM, Bergstralh EJ, Zweig MH, Harris A, Offord KP, Fontana RS. Extended lung cancer incidence follow-up in the Mayo Lung Project and overdiagnosis. J Natl Cancer Inst. 2006;98(11):748-756.

14. Doria-Rose VP, Marcus PM, Szabo E, Tockman MS, Melamed MR, Prorok PC. Randomized controlled trials of the efficacy of lung cancer screening by sputum cytology revisited: a combined mortality analysis from the Johns Hopkins Lung Project and the Memorial Sloan-Kettering Lung Study. Cancer. 2009;115(21):5007-5017.

15. Melamed MR, Flehinger BJ, Zaman MB, Heelan RT, Perchick WA, Martini N. Screening for early lung cancer. Results of the Memorial Sloan-Kettering study in New York. Chest. 1984;86(1):44-53.

16. Tockman MS. Survival and mortality from lung cancer in a screened population: the Johns Hopkins study. Chest. 1986; 89(suppl):324S-325S.

17. Tockman MS, Levin ML, Frost JK, et al. Screening and detection of lung cancer. In: Aisner J, ed. Lung Cancer: Contemporary Issues in Clinical Oncology. New York, NY: Churchill-Livingstone; 1985:25-40.

18. Fontana RS, Sanderson DR, Woolner LB, et al. Screening for lung cancer. A critique of the Mayo Lung Project. Cancer. 1991;67(suppl 4):1155-1164.

19. Kubík A, Parkin DM, Khlat M, Erban J, Polak J, Adamec M. Lack of benefit from semi-annual screening for cancer of the lung: follow-up report of a randomized controlled trial on a population of high-risk males in Czechoslovakia. Int J Cancer. 1990;45(1):26-33.

20. Marcus PM, Bergstralh EJ, Fagerstrom RM, et al. Lung cancer mortality in the Mayo Lung Project: impact of extended follow-up. J Natl Cancer Inst. 2000;92(16):1308-1316.

21. Friedman GD, Collen MF, Fireman BH. Multiphasic Health Checkup Evaluation: a 16-year follow-up. J Chronic Dis. 1986; 39(6):453-463.

22. Fontana RS. The Mayo Lung Project: a perspective. Cancer. 2000;89(suppl 11):2352-2355.

23. Kubík AK, Parkin DM, Zatloukal P. Czech Study on Lung Cancer Screening: post-trial follow-up of lung cancer deaths up to year 15 since enrollment. Cancer. 2000;89(suppl 11): 2363-2368.

24. Brett GZ. The value of lung cancer detection by six-monthly chest radiographs. Thorax. 1968;23(4):414-420.

25. Hocking WG, Hu P, Oken MM, et al; PLCO Project Team. Lung cancer screening in the randomized Prostate, Lung, Colorectal, and Ovarian (PLCO) Cancer Screening Trial. J Natl Cancer Inst. 2010;102(10):722-731.

26. Aberle DR, Adams AM, Berg CD, et al; National Lung Screening Trial Research Team. Reduced lung-cancer mortality with low-dose computed tomographic screening. $N$ Engl J Med. 2011;365(5):395-409.

27. Blanchon T, Bréchot JM, Grenier PA, et al; Dépiscan Group. Baseline results of the Depiscan study: a French randomized pilot trial of lung cancer screening comparing low dose CT scan (LDCT) and chest X-ray (CXR). Lung Cancer. 2007; 58(1):50-58.

28. Garg K, Keith RL, Byers T, et al. Randomized controlled trial with low-dose spiral CT for lung cancer screening: feasibility study and preliminary results. Radiology. 2002;225(2): 506-510.

29. Gohagan JK, Marcus PM, Fagerstrom RM, et al; LUNG SCREENING STUDY RESEARCH GROUP. Final results of the Lung Screening Study, a randomized feasibility study of spiral CT versus chest X-ray screening for lung cancer. Lung Cancer. 2005;47(1):9-15.

30. Infante M, Cavuto S, Lutman FR, et al; DANTE Study Group. A randomized study of lung cancer screening with spiral computed tomography: three-year results from the DANTE trial. Am J Respir Crit Care Med. 2009;180(5): 445-453.

31. Lopes Pegna A, Picozzi G, Mascalchi M, et al; ITALUNG Study Research Group. Design, recruitment and baseline results of the ITALUNG trial for lung cancer screening with low-dose CT. Lung Cancer. 2009;64(1):34-40.

32. van Klaveren RJ, Oudkerk M, Prokop M, et al. Management of lung nodules detected by volume CT scanning. $N$ Engl J Med. 2009;361(23):2221-2229.

33. Saghir Z, Dirksen A, Ashraf H, et al. CT screening for lung cancer brings forward early disease. The randomised Danish Lung Cancer Screening Trial: status after five annual screening rounds with low-dose CT. Thorax. 2012;67(4): 296-301.

34. Bastarrika G, García-Velloso MJ, Lozano MD, et al. Early lung cancer detection using spiral computed tomography and positron emission tomography. Am J Respir Crit Care Med. 2005;171(12):1378-1383

35. Henschke CI, Naidich DP, Yankelevitz DF, et al. Early lung cancer action project: initial findings on repeat screenings. Cancer. 2001;92(1):153-159.

36. Menezes RJ, Roberts HC, Paul NS, et al. Lung cancer screening using low-dose computed tomography in at-risk individuals: the Toronto experience. Lung Cancer. 2010; 67(2):177-183. 
37. Pastorino U, Bellomi M, Landoni C, et al. Early lung-cancer detection with spiral CT and positron emission tomography in heavy smokers: 2-year results. Lancet. 2003;362(9384): 593-597.

38. Sobue T, Moriyama N, Kaneko M, et al. Screening for lung cancer with low-dose helical computed tomography: antilung cancer association project. J Clin Oncol. 2002;20(4): 911-920.

39. Swensen SJ, Jett JR, Hartman TE, et al. CT screening for lung cancer: five-year prospective experience. Radiology. 2005;235(1):259-265.

40. Veronesi G, Bellomi M, Mulshine JL, et al. Lung cancer screening with low-dose computed tomography: a non-invasive diagnostic protocol for baseline lung nodules. Lung Cancer. 2008;61(3):340-349.

41. Wilson DO, Weissfeld JL, Fuhrman CR, et al. The Pittsburgh Lung Screening Study (PLuSS): outcomes within 3 years of a first computed tomography scan. Am J Respir Crit Care Med. 2008;178(9):956-961.

42. Callol L, Roig F, Cuevas A, et al. Low-dose CT: a useful and accessible tool for the early diagnosis of lung cancer in selected populations. Lung Cancer. 2007;56(2):217-221.

43. Diederich S, Thomas M, Semik M, et al. Screening for early lung cancer with low-dose spiral computed tomography: results of annual follow-up examinations in asymptomatic smokers. Eur Radiol. 2004;14(4):691-702.

44. MacRedmond R, McVey G, Lee M, et al. Screening for lung cancer using low dose CT scanning: results of 2 year follow up. Thorax. 2006;61(1):54-56.

45. Novello S, Fava C, Borasio P, et al. Three-year findings of an early lung cancer detection feasibility study with low-dose spiral computed tomography in heavy smokers. Ann Oncol. 2005;16(10):1662-1666.

46. Picozzi G, Paci E, Lopez Pegna A, et al. Screening of lung cancer with low dose spiral CT: results of a three year pilot study and design of the randomised controlled trial "Italung-CT". Radiol Med (Torino). 2005;109(1-2):17-26.

47. Saghir Z, Ashraf H, Dirksen A, Brodersen J, Pedersen JH. Contamination during 4 years of annual CT screening in the Danish Lung Cancer Screening Trial (DLCST). Lung Cancer. 2011;71(3):323-327.

48. Aberle DR, Berg CD, Black WC, et al; National Lung Screening Trial Research Team. The National Lung Screening Trial: overview and study design. Radiology. 2011;258(1):243-253.

49. Mascalchi M, Mazzoni LN, Falchini M, et al. Dose exposure in the ITALUNG trial of lung cancer screening with low-dose CT. Br J Radiol. 2012;85(1016):1134-1139.

50. American Association of Physicists in Medicine. AAPM Position Statement on Radiation Risks from Medical Imaging Procedures.. College Park, MD: American Association of Physicists in Medicine; 2011. Policy PP25-A.

51. Charles M. UNSCEAR report 2000: sources and effects of ionizing radiation. United Nations Scientific Committee on the Effects of Atomic Radiation. J Radiol Prot. 2001;21(1):83-86.

52. International Commission on Radiological Protection. Radiological protection and safety in medicine: ICRP Publication 73. Ann ICRP. 1996;26(2):1-31.

53. Einstein AJ, Henzlova MJ, Rajagopalan S. Estimating risk of cancer associated with radiation exposure from 64-slice computed tomography coronary angiography. JAMA. 2007;298(3): 317-323.

54. Preston DL, Ron E, Tokuoka S, et al. Solid cancer incidence in atomic bomb survivors: 1958-1998. Radiat Res. 2007;168(1):1-64.

55. Bach PB, Kattan MW, Thornquist MD, et al. Variations in lung cancer risk among smokers. J Natl Cancer Inst. 2003; 95(6):470-478.
56. Memorial Sloan-Kettering Cancer Center. Prediction tools. 2003. MemorialSloan-Kettering CancerCenterwebsite.http:// www.mskcc.org/mskcc/html/12463.cfm. Accessed August 12, 2012.

57. Clarke MG, Kennedy KP, MacDonagh RP. Development of a clinical prediction model to calculate patient life expectancy: the measure of actuarial life expectancy (MALE). Med Decis Making. 2009;29(2):239-246.

58. Tan A, Kuo YF, Goodwin JS. Integrating age and comorbidity to assess screening mammography utilization. Am J Prev Med. 2012;42(3):229-234.

59. Wood DE, Eapen GA, Ettinger DS, et al. Lung cancer screening. J Natl Compr Canc Netw. 2012;10(2):240-265.

60. Aberle DR, Adams AM, Berg CD, et al; National Lung Screening Trial Research Team. Baseline characteristics of participants in the randomized national lung screening trial. J Natl Cancer Inst. 2010;102(23):1771-1779.

61. Bach PB, Jett JR, Pastorino U, Tockman MS, Swensen SJ, Begg CB. Computed tomography screening and lung cancer outcomes. JAMA. 2007;297(9):953-961.

62. Wiener RS, Schwartz LM, Woloshin S, Welch HG. Populationbased risk for complications after transthoracic needle lung biopsy of a pulmonary nodule: an analysis of discharge records. Ann Intern Med. 2011;155(3):137-144.

63. Infante M, Chiesa G, Solomon D, et al; DANTE Study Group. Surgical procedures in the DANTE trial, a randomized study of lung cancer early detection with spiral computed tomography: comparative analysis in the screening and control arm. $J$ Thorac Oncol. 2011;6(2):327-335.

64. Petersen RH, Hansen HJ, Dirksen A, Pedersen JH. Lung cancer screening and video-assisted thoracic surgery. J Thorac Oncol. 2012;7(6):1026-1031.

65. Boffa DJ, Allen MS, Grab JD, Gaissert HA, Harpole DH, Wright CD. Data from The Society of Thoracic Surgeons General Thoracic Surgery database: the surgical management of primary lung tumors. J Thorac Cardiovasc Surg. 2008; $135(2): 247-254$.

66. Allen MS, Darling GE, Pechet TT, et al; ACOSOG Z0030 Study Group. Morbidity and mortality of major pulmonary resections in patients with early-stage lung cancer: initial results of the randomized, prospective ACOSOG Z0030 trial. Ann Thorac Surg. 2006;81(3):1013-1019.

67. Schipper PH, Diggs BS, Ungerleider RM, Welke KF. The influence of surgeon specialty on outcomes in general thoracic surgery: a national sample 1996 to 2005 . Ann Thorac Surg. 2009;88(5):1566-1572.

68. Park HS, Detterbeck FC, Boffa DJ, Kim AW. Impact of hospital volume of thoracoscopic lobectomy on primary lung cancer outcomes. Ann Thorac Surg. 2012;93(2):372-379.

69. Farjah F, Flum DR, Varghese TK Jr, Symons RG, Wood DE. Surgeon specialty and long-term survival after pulmonary resection for lung cancer. Ann Thorac Surg. 2009;87(4):995-1004.

70. Learn PA, Bach PB. A decade of mortality reductions in major oncologic surgery: the impact of centralization and quality improvement. Med Care. 2010;48(12):1041-1049.

71. Cheng D, Downey RJ, Kernstine K, et al. Video-assisted thoracic surgery in lung cancer resection: a meta-analysis and systematic review of controlled trials. Innovations (Phila). 2007;2(6):261-292.

72. Yan TD, Black D, Bannon PG, McCaughan BC. Systematic review and meta-analysis of randomized and nonrandomized trials on safety and efficacy of video-assisted thoracic surgery lobectomy for early-stage non-small-cell lung cancer. J Clin Oncol. 2009;27(15):2553-2562.

73. Howington JA, Blum MG, Chang AC, Balekian AA, Murthy SC. Treatment of stage I and II non-small cell lung cancer: diagnosis and management of lung cancer, 3rd ed: American 
College of Chest Physicians evidence-based clinical practice guidelines. Chest. 2013;143(5)(suppl):e278S-e313S.

74. Cassidy A, Myles JP, van Tongeren M, et al. The LLP risk model: an individual risk prediction model for lung cancer. Br J Cancer. 2008;98(2):270-276.

75. Spitz MR, Hong WK, Amos CI, et al. A risk model for prediction of lung cancer. J Natl Cancer Inst. 2007;99(9):715-726.

76. D’Amelio AM Jr, Cassidy A, Asomaning K, et al. Comparison of discriminatory power and accuracy of three lung cancer risk models. Br J Cancer. 2010;103(3):423-429.

77. Etzel CJ, Bach PB. Estimating individual risk for lung cancer. Semin Respir Crit Care Med. 2011;32(1):3-9.

78. Spitz MR, Etzel CJ, Dong Q, et al. An expanded risk prediction model for lung cancer. Cancer Prev Res (Phila). 2008;1(4): 250-254.

79. Tammemagi CM, Pinsky PF, Caporaso NE, et al. Lung cancer risk prediction: Prostate, Lung, Colorectal And Ovarian Cancer Screening Trial models and validation. J Natl Cancer Inst. 2011;103(13):1058-1068.

80. Raji O, Duffy S, Olorunshola A, et al. Predictive accuracy and clinical utility of the Liverpool Lung Project Risk Model using population-based data from Europe and North America In: Proceedings from the National Cancer Research Institute; November 7-11, 2010; Liverpool, England. Abstract LB24A.

81. Silvestri GA, Nietert PJ, Zoller J, Carter C, Bradford D. Attitudes towards screening for lung cancer among smokers and their non-smoking counterparts. Thorax. 2007;62(2):126-130.
82. Patel D, Akporobaro A, Chinyanganya N, et al; LungSEARCH Investigators. Attitudes to participation in a lung cancer screening trial: a qualitative study. Thorax. 2012;67(5): 418-425.

83. Detterbeck FC, Mazzone PJ, Naidich DP, Bach PB. Screening for lung cancer: diagnosis and management of lung cancer, 3rd ed: American College of Chest Physicians evidencebased clinical practice guidelines. Chest. 2013;143(5)(suppl): e78S-e92S.

84. Wender R, Fontham ET, Barrera E Jr, et al. American Cancer Society lung cancer screening guidelines [published online ahead of print January 11, 2013]. CA Cancer J Clin. 2013. doi:10.3322/caac. 21172 .

85. Field JK, Smith RA, Aberle DR, et al; IASLC CT Screening Workshop 2011 Participants. International Association for the Study of Lung Cancer Computed Tomography Screening Workshop 2011 report. J Thorac Oncol. 2012;7(1):10-19.

86. Jaklitsch MT, Jacobson FL, Austin JHM, et al. The American Association for Thoracic Surgery guidelines for lung cancer screening using low-dose computed tomography scans for lung cancer survivors and other high-risk groups. J Thorax Cardiovasc Surg. 2012;144:33-38.

87. Rocco G, Allen M, Altorki N, et al. Clinical statement on the role of the surgeon and surgical issues relating to CT screening programs for lung cancer: Report from The Society of Thoracic Surgeons CT Screening for Lung Cancer Task Force. Ann Thorac Surg. In press. 